\title{
Comparison of GeneXpert against Light-Emitting Diode Fluorescent Microscopy for the Diagnosis of Pulmonary Tuberculosis in Addis Ababa, Ethiopia
}

Boja Dufera Taddese ${ }^{1 *}$, Daniel Melese Desalegn ${ }^{1}$, Abay Sisay Misganaw², Kumera Terfa Kitila ${ }^{1}$, Hanna Mekonnen Balcha ${ }^{1}$, Chalachew Sisay Gebeyehu', Tinsae Kidanemariam Hailu ${ }^{1}$ and Abraham Tesfaye Bika ${ }^{1}$

${ }^{1}$ Addis Ababa Health Bureau, Public Health Research and Emergency Management, Addis Ababa, Ethiopia

${ }^{2}$ Department of Medical Laboratory, School of Allied Health sciences, College of Health Sciences, Addis Ababa University, Addis Ababa, Ethiopia

\begin{abstract}
Background: Tuberculosis remains a global health problem despite availability of effective tools. Globally, there were $40 \%$ of the $10+$ million patients with TB did not get diagnosed or notified. The study was aimed to compare the diagnostic performances of iLED-FM and GeneXpert test for the diagnosis of PTB in Addis Ababa, Ethiopia.

Methods: Facility-based cross-sectional study was conducted on a total of 286 sputum samples collected from health centers and hospitals clients with presumptive TB from December 2016 to March 2017. Kappa value, Sensitivity, Specificity, Positive Predictive Value (PPV) and Negative Predictive Value (NPV) of iLED-FM and GeneXpert were calculated against the gold standard.

Results: The sensitivity, specificity, PPV and NPV of iLED-FM was $80.15 \%, 95.48 \%, 93.75 \%$ and $85.06 \%$, for GeneXpert $88.55 \%, 92.90 \%, 91.34 \%$ and $90.57 \%$, respectively. Kappa value of iLED-FM was 0.765 and 0.817 for GeneXpert. Out of 55 HIV positive presumptive TB patients enrolled in the study, 19 (34.55\%) were sputum smear positive by iLED-FM. However, 24 (43.64\%) TB cases were detected by GeneXpert.
\end{abstract}

Conclusion: The sensitivity of Xpert MTB/RIF assay was better than iLED-FM in the diagnosis of PTB. It should be implemented as primary diagnostic test in areas where overlapping synergy of TB and HIVIAIDS is high.

Keywords: Addis Ababa; Ethiopia; GeneXpert; Light-emitting diode; Microscopy; Tuberculosis

\begin{abstract}
Abbreviations: AIDS: Acquired Immuno Deficiency Virus; CI: Confidence Interval; HIV: Human Immuno-Virus; LED-FM: Light Emitting Diode Fluorescent Microscopy; L-J: Lowenstein-Jensen Media; MTB: Mycobacterium tuberculosis; MGIT: Mycobacterial Growth Indicator Tube; MDR-TB: Multi-Drug Resistance Tuberculosis; PPV: Positive Predictive Value; NPV: Negative Predictive Value; SOPs: Standard Operating Procedures; SPSS: Statistical Package for Social Science; TB: Tuberculosis; WHO: World Health Organization
\end{abstract}

\section{Introduction}

Despite availability of effective tools and the main advances in TB diagnostic technologies over the world, $\mathrm{ZN}$-stained direct sputum smear microscopy remains the most widely used diagnostic method available in most health-care laboratories of developing countries [1]. However, it has low sensitivity compared to auramine-O stained sputum smear examined by LED-FM and Mycobacterium tuberculosis (MTB) culture $[1,2]$. It is a responsibility for diagnosing of $40 \%$ of the $10+$ million patients with TB did not get diagnosed or notified in globally $[3,4]$.

Conventional culture has been estimated to detect 10 to 100 viable MTB per milliliter of sputum [3]. But this method requires infrastructures, costly, technical demanding, time taking for result delivery and not suitable for patient management $[1,2]$.

Recently, molecular diagnostics are increasingly being promoted as TB diagnostic tools due to rapid turnaround time, high sensitivity and specificity of the techniques [2,5]. Consequently, WHO recommend GeneXpert to be used as an initial diagnostic test in individuals suspected of Multi-Drug resistance tuberculosis (MDR-TB), extra PTB, HIV-associated TB and children suspected of TB. Children and HIV/AIDS patients are immune compromised and unable to spout out productive sputum. Hence, misdiagnosis is common by $\mathrm{ZN}$-techniques in these patient groups [6].

In Ethiopia, most health facilities have limited capacities to diagnose TB. For instance, in 59\% health facilities in Ethiopia, the only available diagnostic test was sputum smear microscopy, $6 \%$ have chest $\mathrm{x}$-ray and $2 \%$ have GeneXpert. According to center for strategic and international studies, nearly $48 \%$ of all active TB cases would have been missed while screened by sign and symptoms [7]. Hence, the need to expand and utilize different diagnostic modalities in different context of Ethiopia plays great role in increasing case detection and control of TB.

In study setting, there is a direction to utilize GeneXpert for all TB presumptive patients' diagnosis. However, in Ethiopia, only $2 \%$ health facilities have GeneXpert machine to diagnose TB [6]. As a result, access limits its utilization for all TB presumptive patients. Hence, there is greater need of other alternative diagnostic methods particularly in study area and other similar settings.

Even though WHO recommended LED-FM be used as an alternative to $\mathrm{ZN}$-stained sputum smear microscopy for $\mathrm{TB}$ case detection, the

*Corresponding author: Boja Dufera T, Addis Ababa City Administration Health Bureau, Public Health Research and Emergency Management, Ethiopia, Tel: 251920343550; E-mail: duferataddese@yahoo.com

Received January 19, 2018; Accepted February 10, 2018; Published February 17, 2018

Citation: Taddese BD, Desalegn DM, Misganaw AS, Kitila KT, Balcha HM, et al. (2018) Comparison of GeneXpert against Light-Emitting Diode Fluorescent Microscopy for the Diagnosis of Pulmonary Tuberculosis in Addis Ababa, Ethiopia. J Microb Biochem Technol 10: 12-15. doi: 10.4172/1948-5948.1000388

Copyright: @ 2018 Taddese BD, et al. This is an open-access article distributed under the terms of the Creative Commons Attribution License, which permits unrestricted use, distribution, and reproduction in any medium, provided the original author and source are credited. 
Citation: Taddese BD, Desalegn DM, Misganaw AS, Kitila KT, Balcha HM, et al. (2018) Comparison of GeneXpert against Light-Emitting Diode Fluorescent Microscopy for the Diagnosis of Pulmonary Tuberculosis in Addis Ababa, Ethiopia. J Microb Biochem Technol 10: 12-15. doi: $10.4172 / 1948-5948.1000388$

diagnostic performance of LED-FM was not evaluated in study setting [1]. Therefore, this study was aimed to compare the performance of auramine $\mathrm{O}$-stained direct sputum smear examined by LED-FM against GeneXpert test for the diagnosis of PTB in Addis Ababa, Ethiopia.-

\section{Materials and Methods}

\section{Study design and setting}

A facility based cross-sectional study design was conducted from December 2016 to March 2017 to compare the diagnostic performances of iLED-FM and GeneXpert test for the diagnosis of PTB in Addis Ababa, Ethiopia. Based on 2007 census conducted by Ethiopian national statistics authorities, Addis Ababa has 10 sub city and 116 Woreda (the lowest District of City Administration) with an estimated total population of 3,384,569 and annual growth rate of 3.8\% [8]. In the city, there are more 896 public and private health facilities, of which 101 public and 52 privates are being delivering TB diagnostic services [9]. Sputum specimens are referred though postal system to regional laboratory or lead hospitals where Xpert MTB/RIF assay machines are available and result sent back to referred health facilities through postal system of the existing national referral linkage map of testing network.

\section{Sample size determination and sampling techniques}

The sample size was determined using single population proportion formula considering the crude prevalence of TB in Ethiopia as 19\% for convenience [10]. Accordingly, a total of 286 sputum samples were collected from TB presumptive patients from private and public health facilities of Addis Ababa during the study period were included.

Laboratory testing procedures and sputum specimen management were performed according to stated Standard Operating Procedures (SOPs) of LED-FM sputum smear microscopy, GeneXpert and L-J sputum culture $[3,6]$. Seven to ten milliliters of sputum samples $(2-4$ $\mathrm{ml}$ for GeneXpert and the remaining for culture) were collected in two separate falcon tubes. Strict procedures were followed during reagents preparation, sputum smears preparation and staining. The qualities of the prepared reagents were checked by staining with known positive and negative quality control sputum smear slides. After quality checks of the reagents, smears prepared from direct sputum were stained by auramine-O, air dried and examined by LED-FM [11-13].

Before inoculation on L-J culture media, sputum samples were decontaminated by N-Acetlyl-L-Cysteine-Sodium Hydroxide (NALC$\mathrm{NaOH}$ ) [3]. The sterility of the prepared culture media were checked by incubating in an incubator for $48 \mathrm{~h}$ at $37^{\circ} \mathrm{C}$ to see if there is no over growth of other contaminants [6]. After quality check of the prepared culture media, $0.1 \mathrm{ml}$ of decontaminated and distilled water re suspended sputum sample was inoculated on (L-J) media [3].The other non-decontaminated sputum samples in the second falcon tubes were analyzed by GeneXpert test [6].

Sputum processing for smear preparations and liquefaction for GeneXpert test were performed in bio-safety level-2 laboratories dedicated for MTB work with an appropriate ventilation system. Decontamination and inoculation on L-J culture media were performed in bio-safety level-3 and accesses to the room were restricted when the work was in progress. Appropriate protective equipment including gowns, gloves and respirator masks (N-95) were used while handling specimens [3]. Before removing from laboratory, all tubes with the culture media were sterilized using an autoclave at $121^{\circ} \mathrm{C}$ for $15 \mathrm{~min}$ to the acceptable level of sterilization [3].

\section{Data management and statistical analysis}

The results of each test were entered to the excel sheet, checked for completeness, consistency and transferred to SPSS version 20 (SPSS Inc. Chicago, USA) software for analyses. The sensitivity, specificity, PPV and NPV of LED-FM and GeneXpert were calculated against the gold standard.

\section{Results}

\section{Socio-demographic characterstics of study participants}

From the total of 286 PTB presumptive patients enrolled in the study, $117(40.91 \%)$ were female. The average age of the study participants were 35.13 year with the minimum and maximum age of 18 and 80 , respectively. Of 286 study participants, $172(60.14 \%)$ were tested for HIV/AIDS of whom male accounted 96 (55.81\%). Sero-positivity rate among those tested for HIV/AIDS were 55 (32\%); 26 (27.08\%) among male and $29(38.16 \%)$ among female as illustrated in Table 1, of 55 sero positives, $24(43.6 \%)$ were positive for TB by GeneXpert test but 19 (34.5\%) were positive by LED-FM as presented in Table 2 .

On the other hand, treatment categories of patients screened for MTB were reviewed from the requested paper. Of total the PTB presumptive patients enrolled in the study, 148 (51.75\%) were new cases, $73(25.52 \%)$ relapse, $22(7.70 \%)$ treatment failure, $1(0.35 \%)$ defaulter and $41(14.34 \%)$ have no recorded previous treatment history.

\section{Comparison of direct LED-FM and culture results}

From a total of 286 sputum samples analyzed by culture and LEDFM, 105 (36.71\%) were positive by both methods; 7 (2.45\%) were smear positive by LED-FM but culture negative; $148(51.20 \%)$ were negative by both methods and 26 (9.09\%) were culture positive but smear negative by LED-FM (Table 3). The sensitivity, specificity, PPV and NPV of LED-FM was $80.15 \%$ (95\% CI: 72.29\%-88.61\%), 95.48\% (95\% CI:

\begin{tabular}{|c|c|c|c|}
\hline Age group in years & Male (\%) & Female (\%) & Total (\%) \\
\hline $18-27$ & $43(25.44)$ & $41(35.04)$ & $84(29.37)$ \\
\hline $28-37$ & $62(36.69)$ & $35(29.91)$ & $97(33.92)$ \\
\hline $38-47$ & $31(18.34)$ & $23(19.66)$ & $54(18.88)$ \\
\hline $48-57$ & $15(8.88)$ & $12(10.26)$ & $27(9.44)$ \\
\hline $58-67$ & $12(7.10)$ & $2(1.71)$ & $14(4.90)$ \\
\hline$\geq 68$ & $6(3.55)$ & $4(3.42)$ & $10(3.50)$ \\
\hline Total & $169(59.09)$ & $117(40.91)$ & $286(100)$ \\
\hline HIV Status & & & \\
\hline Positive & $26(26.08)$ & $29(38.16)$ & $55(31.98)$ \\
\hline Negative & $70(72.92)$ & $47(61.84)$ & $117(68.02)$ \\
\hline Total & $96(100)$ & $76(100)$ & $172(100)$ \\
\hline
\end{tabular}

Table 1: Socio-demographic characterstics of study participants by age, sex and HIV-status, Addis Ababa, Ethiopia.

\begin{tabular}{|l|c|c|c|}
\hline & \multicolumn{2}{|c|}{ HIV status } & \\
\hline LED-FM & Positive & Negative & Total \\
\hline Smear positive & 19 & 64 & 83 \\
\hline Smear negative & 36 & 53 & 89 \\
\hline GeneXpert & & & \\
\hline MTB detected & 24 & 50 & 74 \\
\hline MTB not detected & 31 & 67 & 98 \\
\hline MTB culture & & & 69 \\
\hline Positive & 22 & 47 & 103 \\
\hline Negative & 33 & 70 & \\
\hline
\end{tabular}

Table 2: HIV-results and TB results with diagnostic methods, Addis Ababa, Ethiopia. 
Citation: Taddese BD, Desalegn DM, Misganaw AS, Kitila KT, Balcha HM, et al. (2018) Comparison of GeneXpert against Light-Emitting Diode Fluorescent Microscopy for the Diagnosis of Pulmonary Tuberculosis in Addis Ababa, Ethiopia. J Microb Biochem Technol 10: 12-15. doi: $10.4172 / 1948-5948.1000388$

\begin{tabular}{|c|c|c|c|c|}
\hline \multirow{2}{*}{$\begin{array}{l}\text { Methods to be } \\
\text { Evaluated }\end{array}$} & \multicolumn{3}{|c|}{ Mycobacterium Culture } & \multirow[b]{2}{*}{ Total (\%) } \\
\hline & & Positive (\%) & Negative (\%) & \\
\hline \multirow{3}{*}{ iLED-FM } & Positive (\%) & $105(36.71)$ & $7(2.45)$ & $112(39.16)$ \\
\hline & Negative (\%) & $26(9.09)$ & $148(51.75)$ & $174(60.84)$ \\
\hline & Total & $131(45.80)$ & $155(54.20)$ & $286(100)$ \\
\hline \multirow{3}{*}{$\begin{array}{l}\text { Xpert MTB/RIF } \\
\text { assay }\end{array}$} & Positive & $116(40.56)$ & $11(3.85)$ & $127(44.41)$ \\
\hline & Negative & $15(5.24)$ & $144(50.35)$ & $159(59.59)$ \\
\hline & Total & $131(45.80)$ & $155(54.20)$ & $286(100)$ \\
\hline
\end{tabular}

Table 3: Comparison of LED-FM, GeneXpert and culture results, Addis Ababa, Ethiopia.

90.92\%-98.17\%), $93.75 \%$ (95\% CI: $87.86 \%$ to $96.88 \%)$ and $85.06 \%(95 \%$ CI: $80.11 \%$ to $88.94 \%)$, respectively. The diagnostic yeild and accuracy of the test method was 36.71 and 39.16 , respectively. The measure of agreement between the LED-FM and reference standard results were substantial with kappa coeffient $(\mathrm{k}=0.765)$ and $\mathrm{p}$-value $(<0.001)$.

\section{Comparison of GeneXpert and culture results}

From the total of 286 sputum samples analyzed by both GeneXpert and L-J culture, $116(40.56 \%)$ were positive by both methods,11(3.85\%) were positive by GeneXpert but culture negative, 144 (50.35\%) were negative by both GeneXpert and MTB culture while 15 (5.24\%) were negative by GeneXpert but culture positive (Table 3 ). The final results of both GeneXpert and culture were provided to the physician of the patients for the treatement decision. The sensitivity, specificty, PPV and NPV of GeneXpert was $88.55 \%$ (95\% CI: $81.82 \%$ to $93.45 \%$ ), $92.90 \%$ (95\% CI: $87.66 \%$ to $96.40 \%$ ), $91.34 \%$ (95\% CI: $85.60 \%$ to $94.92 \%$ ) and 90.57\% (95\% CI: $85.61 \%$ to $93.93 \%$ ) respectively. The diagnostic yeild and accuracy of the test method was $40.56 \%$ and $44.41 \%$, respectively. The measure of agreement between GeneXpert and culture results were perfect with kappa coeffient $(\mathrm{k}=0.817)$ and $\mathrm{p}$-value $(<0.001)$.

\section{Comparison of LED-FM, GeneXpert and culture results}

As presented in Table 3 from the total of 286 sputum samples examined by LED-FM, GeneXpert and culture, 105 were positive by LED-FM and culture. However, 116 cases were positive by GeneXpert and culture.

\section{Discussion}

In the present study, the diagnostic performances of auramine O-stained sputum smear examined by LED-FM and GeneXpert test were compared against MTB culture as a gold standard. The sensitivity, specificity, PPV value and NPV of GeneXpert was $88.55 \%, 92.90 \%$, $91.34 \% 90.57 \%$, respectively and LED-FM was $80.15 \%, 95.48 \%, 93.75 \%$, $85.06 \%$, respectively.

The sensitivity of GeneXpert in the present study was similar to meta-analyses reports of 22 studies pooled sensitivity $88 \%$ of WHO implementation manual 2014 [11]. In addition, it was in line with the sensitivity $90.1 \%$ reported from Pakistan [14]. However, it was lower compared to $97.14 \%$ reported from Egypt [15]. The difference might be due to from the sample size. Study in Egypt was conducted on 40 sputum samples but the present study, 286 sputum samples were analyzed.

The sensitivity of GeneXpert in current study was higher than the sensitivity $83.9 \%$ reported from Thailand [16]. The study carried out in Thailand was on smear negative PTB while the present study include both smear positive and negative sputum samples which might attributed to the difference. The sensitivity of the present study was higher than the study conducted in Kosovo, sensitivity 82.3\% [17]. The difference might result from the sample size used in the study. Study conducted in Kosovo, 116 sputum samples were analyzed.

The specificity of GeneXpert in the present study was lower than the specificity $98.3 \%$ reported from Pakistan [14]. The difference might be due to sample size, study setting and sample type. In study of Pakistan, a total 403 samples were analyzed of which some are extra pulmonary specimens. The specificity of the current study was higher compared to specificity $86.4 \%$ of study conducted in Thailand [18]. The difference might be due to small sample size (109) and the gold standard used. The in study of Thailand, Mycobacterium Growth Indicator Tube (MGIT) was used as the gold standard, some of which grew non-tuberculosis Mycobacteria.

The specificity of GeneXpert in the current study is higher compared to $75 \%$ of study conducted in Egypt [19]. This might be attributed to study population and sample size. The present study includes all treatment categories and the sample size 286. But the study in Egypt includes retreatment cases and sample size of only fifty eight.

The sensitivity of LED-FM in the present study was higher than 73.2\% reported from Kenya [19]. The study conducted in Kenya used larger sample size (1394) and the prevalence of TB in general population may vary. Our study result showed almost comparable sensitivity with WHO LED microscopy policy implementation $84 \%$ and $84.7 \%$ reported from Cape Town, South Africa [1,20,21]. Meta analyses conducted on 45 studies indicated the sensitivity LED-FM ranged from 52\% to $97 \%$ which encompass the present study sensitivity [22].

In this study, the specificity of LED-FM 95.48\% was comparable to study reported from Kenya specificity $96.7 \%$ [23]. The specificity of our study finding was relatively comparable with WHO LED microscopy policy implementation $98 \%$ and $98.9 \%$ of study reported from Cape Town, South Africa [1].

\section{Conclusion and Recommendation}

In this study, the sensitivity of GeneXpert is better than LED-FM for the diagnosis of PTB. It should be implemented as primary diagnostic test in areas where overlapping synergy of TB and HIV/AIDS is high. The implementation of LED-FM should be supplemented by GeneXpert in areas where the prevalence of HIV/AIDS is high. For sero-negative presumptive TB patients LED-FM should be implemented as diagnostic test of PTB in Addis Ababa, Ethiopia.

\section{Acknowledgment}

The authors would like to thank Addis Ababa City Administration Health Bureau and health facilities in the city for their cooperation. We would like to thank medical laboratory professionals in these health facilities for collecting the sputum samples post-men who brought the samples to Addis Ababa regional laboratory for analysis.

\section{References}

1. Pai M (2018) A free TB resource for primary care providers. Nat Microbiol.

2. WHO (2011) Fluorescent light-emitting diode (LED) microscopy for diagnosis of tuberculosis. Policy Statement, pp: 1-5.

3. Alvarez-Uria G, Azcona JM, Midde M, Naik PK, Reddy S, et al. (2012) Rapid diagnosis of pulmonary and extra pulmonary tuberculosis in HIV-infected patients: Comparison of LED fluorescent microscopy and the GeneXpert MTB/ RIF assay in a district hospital in India. Tuber Res Treat, pp: 1-4.

4. Kent BD, Kubica GB (1985) Public Health Mycobacteriology: A Guide for the Level III Laboratory. US Department of Health and Human Service, Center of Disease Controls, Atlanta.

5. Boehme CC, Nabeta P, Hillemann D, Nicol MP, Shenai S, et al. (2010) Rapid molecular detection of tuberculosis and rifampin resistance. $\mathrm{N}$ Eng $\mathrm{J}$ Med 363:1005-1015.

6. World Health Organization (2014) Xpert MTB/RIF implementation manual technical and operational 'how-to'. Practical considerations, pp: 1-42. 
Citation: Taddese BD, Desalegn DM, Misganaw AS, Kitila KT, Balcha HM, et al. (2018) Comparison of GeneXpert against Light-Emitting Diode Fluorescent Microscopy for the Diagnosis of Pulmonary Tuberculosis in Addis Ababa, Ethiopia. J Microb Biochem Technol 10: 12-15. doi: 10.4172/1948-5948.1000388

7. Raves R, Angelo S, Nieburg (2016) As Ethiopia moves toward tuberculosis elimination, success requires higher investment. Center for Strategic and International Studies (CSIS), pp: 1-34

8. Central Statistic Agency of Ethiopia (CSA) (2008) Summary and statistical report of the 2007 population and housing census. Addis Ababa, Ethiopia.

9. Addis Ababa City Administration Health Bureau (2017) TBL, TB/HIV and MDRTB prevention and control program: The $12^{\text {th }}$ TRAC and $20^{\text {th }}$ World TB Day. Addis Ababa, Ethiopia.

10. World Health Organization (2014) Global tuberculosis report. Geneva, Switzerland.

11. Federal Ministry of Health and Ethiopian Public Health Institute (2014) AFB smears microscopy manual. Addis Ababa, Ethiopia.

12. Abay Sisay M, Mulugeta Tsegaye A, Adino Desale L, Abrham Tesfaye B (2016) External quality assessment (EQA) of randomly blinded rechecking slides on TB AFB microscopy laboratories: A retrospective study, Addis Ababa, Ethiopia. Am J Lab Med 1: 9-15.

13. The Global Laboratory Initiative Working Group of the Stop TB Partnership. Mycobacteriology Laboratory Manual, pp: 1-147.

14. Munir MK, Rehman S, Aasim M, Iqbal R, Saeed S (2015) Comparison of Zieh Neelsen microscopy with GeneXpert detection of Mycobacterium tuberculosis. OSR-JDMS 14: 56-60.

15. Darwish M, El Wadood MA, ALnagdis H (2013) Diagnostic assessment of Xpert MTB/RIF in a sample of Mycobacterium tuberculosis Egyptian patients. Afr J Microbiol Res 7: 5107-5113
16. Reechaipichitkul W, Phetsuriyawong A, Chaimanee P, Ananta P (2016) Diagnostic test of sputum GeneXpert MTB/RIF for smear negative pulmonary tuberculosis. Southeast Asian J Trop Med Public Health 47: 475-466.

17. Bajrami R, Mulliqi G, Kurti A, Lila G, Raka L (2016) Comparison of GeneXpert MTB/RIF and conventional methods for the diagnosis of tuberculosis in Kosovo. J Infect Dev Countries 10: 418-422.

18. Pinyopornpanish K, Keawvichit R, Supparatpinyo K, Chaiwarith $R$ Wongworapat K, et al. (2015) Comparison of Xpert MTB/RIF assay and the conventional sputum microscopy in detecting mycobacterium tuberculosis in northern Thailand. Tuber Res Treat, pp: 1-6

19. Meawed TE, Shaker A (2016) Assessment of diagnostic accuracy of GeneXpert MTB/RIF in diagnosis of suspected retreatment pulmonary tuberculosis patients. Egypt J Chest Dis Tub 65: 637-641.

20. Bonnet M, Gagnidze L, Githui W, Guerin PJ, Bonte L, et al. (2011) Performance of LED-based fluorescence microscopy to diagnose tuberculosis in a peripheral health centre in Nairobi. PLoS One 6: 1-6.

21. Marais BJ (2008) Use of light-emitting diode fluorescence microscopy to detect acid-fast Bacilli in sputum. Clin Infect Dis 47: 203-207.

22. Bonnet M, Gagnidze L, Guerin PJ, Bonte L, Ramsay A, et al. (2011) Evaluation of combined LED-fluorescence microscopy and bleach sedimentation for diagnosis of tuberculosis at peripheral health service level. PLoS One 6: 1-7.

23. Steingart KR, Hopewell PC, Ramsay A, Cunningham J, Urbanczik R, et al. (2006) Fluorescence versus conventional sputum smears microscopy for tuberculosis: A systematic review. Lancet Infect Dis 6: 570-581. 